\title{
Metabolism of the myocardium at cellular level
}

\author{
By P. Harris, Institute of Cardiology, University of London, 2 Beaumont Street, \\ London $W_{\mathbf{I}} N_{2} D X$
}

The contractile properties of the heart are the result of the interaction of a complex system of intracellular filaments (Gergely, 1966). These lie longitudinally in the cell and consist of alternating arrays of thick and thin filaments. The thin filaments arise from each side of transversely oriented $Z$ lines and interdigitate with the thick filaments forming a structure which repeats along the length of the cell (Pl. I). The filamentous systems are grouped together in fascicles called myofibrils. Contraction consists of a sliding movement between the thin and thick filaments (Huxley, 1959).

The process of contraction requires energy in the form of the high-energy terminal phosphate grouping of adenosine triphosphate (ATP). Lying alongside the myofibrils are rows of mitochondria which are the intracellular site of production of ATP. Mitochondria are sac-like bodies composed of an outer membrane and an inner membrane which is folded into cristae. It is on the infolded inner membrane that ATP regeneration occurs. This is primarily the function of a system of enzymes, known as the respiratory chain, which convert the energy released by the combustion of hydrogen and oxygen to form water into a biologically useful form. In this process A'TP is regenerated from adenosine diphosphate (ADP) and inorganic phosphate. ATP molecules thus formed can then diffuse to various parts of the cell where their energy can be liberated on site by numerous specific ATPase enzymes which split the ATP molecule into ADP and inorganic phosphate. Such enzymes link the liberation of energy to specific cellular functions. For instance, myofibrillar ATPase provides the energy for contraction of the myofibril.

There is, therefore, a continual recycling of ADP, inorganic phosphate and ATP molecules. The activity of the respiratory chain is governed by the supply of ADP as well as the supply of oxygen and hydrogen so that the regeneration of ATP is to a large extent self-regulatory (Chance \& Williams, I956). An immediate reserve of high-energy phosphate groups is held available in the form of creatine phosphate which can readily transfer its high-energy phosphate to ADP under the influence of the enzyme creatine kinase $(E C$ 2.7.3.3).

The supply of hydrogen to the respiratory chain derives from the classical processes of catabolism of carbohydrate and fat. Complex though these pathways may be, the one fundamental principle which underlies them is the process of 'oxidation' by the removal of hydrogen. The hydrogen which is eliminated is transferred to the respiratory chain by coenzymes, notably nicotine adenine dinucleotide (NAD). 
According to the position in the respiratory chain at which hydrogen enters, two or three molecules of ATP are regenerated for each atom of hydrogen oxidized. We have been brought up to believe that life depends on the combustion of foodstuffs to water and carbon dioxide. This statement is not strictly true. The flame of life is a hydrogen flame; carbon dioxide is a by-product.

The myocardium can utilize a variety of metabolites (Harris, Jones, Bateman, Chlouverakis \& Gloster, I964). Quantitatively, free fatty acids and glucose are the most important. The degree of oxygen extraction by the myocardium is high, the coronary sinus oxygen saturation being in the region of $20 \%$. The tissue itself is well adapted to a high rate of utilization of oxygen, with a rich capillary network and an abundance of mitochondria.

We now know that the heart is not a syncytium but an assembly of individual cells. There is complete separation at the intercalated discs. The properties of the membrane which invest each cell have an important influence on the exchange of ions and determine the excitability of the cell. The membrane is, under resting conditions, relatively impermeable to sodium ions, but permeable to potassium ions. The membrane is the site of action of the sodium pump mechanism which actively expels sodium from the cell in exchange for potassium. In this way the intracellular concentration of sodium is low and that of potassium is high. The action of the sodium pump, together with the different permeabilities of ions, gives rise to the resting transmembrane potential, the inside of the cell being some $80 \mathrm{mV}$ negative with respect to the outside. When excitation occurs the membrane becomes momentarily permeable to sodium ions which move inwards along an electrochemical gradient bearing their positive charge. Thus the transmembrane potential disappears and even for a brief interval is reversed. This is followed by an isoelectric plateau which is terminated by the redevelopment of the resting potential at a time when potassium ions move out of the cell. During the rest of the cardiac cycle the sodium pump has to make good the loss of potassium and gain of sodium associated with the action potential. Since the sodium pump does work moving ions against electrochemical gradients it requires energy. The energy is provided by a specific sodiumpotassium stimulated ATPase present in the cell wall membrane (Gibson \& Harris, I970). This enzyme is specifically inhibited by cardiac glycosides and may, indirectly, form the basis of their action on the heart.

The excitatory wave is thought to pass rapidly into the centre of the cell down a series of transverse microtubules which are located at the level of each $Z$ line. The transverse tubules come into contact with a separate system of longitudinal tubules which invest the myofibrils (Pl. I).

The link between excitation and contraction is mediated by calcium ions (Harris \& Opie, 1971). In the resting state, calcium ions are actively accumulated by the longitudinal tubular system so that the concentration in the cytoplasm is low. The excitatory wave passing down the transverse tubules is thought to cause the longitudinal tubules to liberate their stored calcium ions which then diffuse rapidly into the region of the contractile filaments.

The thick filaments are composed of myosin molecules, the enlarged heads of 


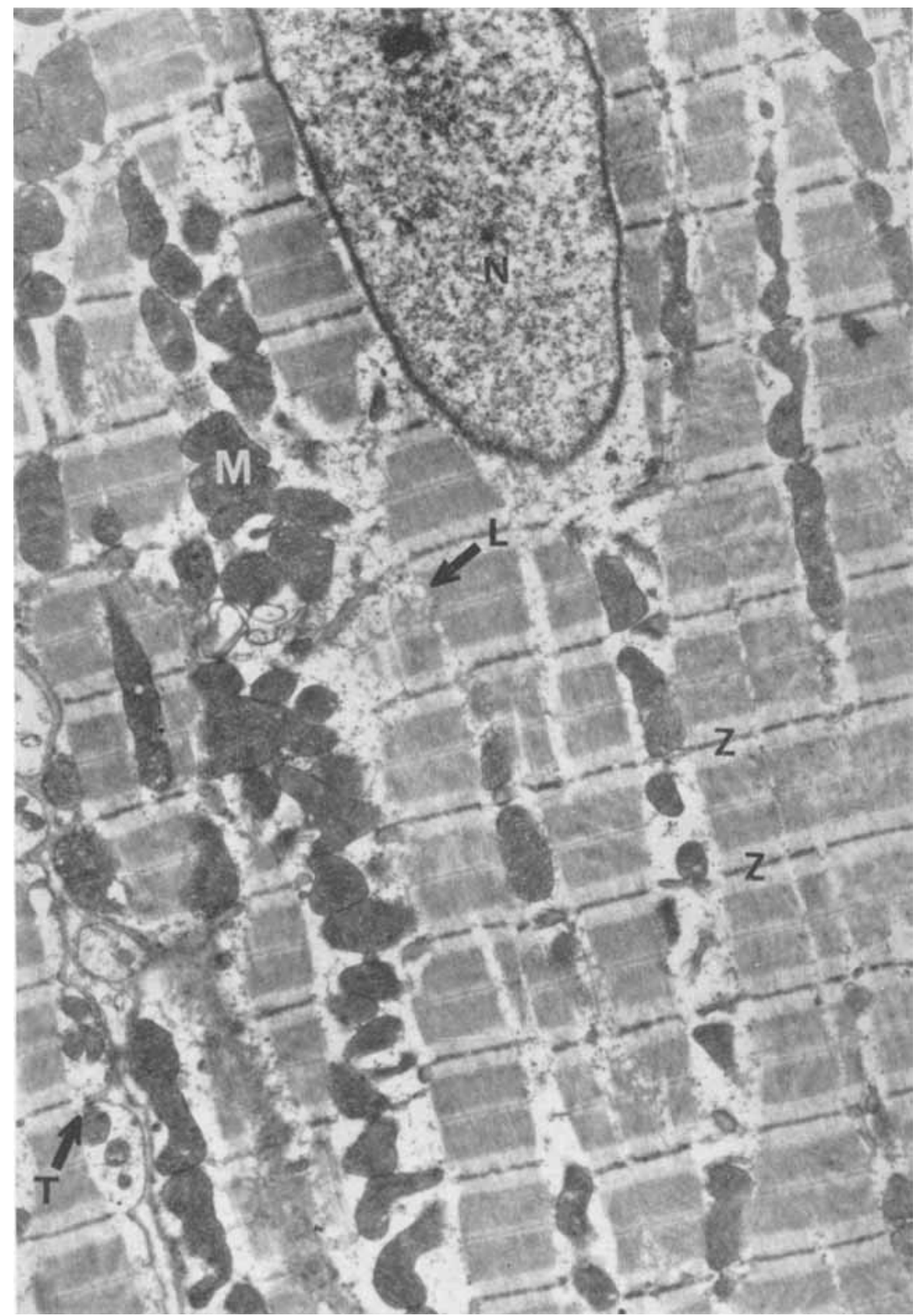

\section{P. HARRIS}

(Facing p. 309) 
which protrude at intervals from the filament. The main component of the thin filaments is actin. Contraction is caused by bridges which develop between the myosin and actin molecules. In the resting state the formation of such bridges is prevented by the presence of the regulatory protein, troponin. This action of troponin is, however, itself inhibited by calcium ions. Thus, when calcium ions are liberated in the vicinity of the myofibril by the excitatory wave, the action of troponin is inhibited and bridges are allowed to form between myosin and actin.

This brief summary reviews the general background of modern studies of the metabolism of the myocardium, in which the minute anatomy of the cell is as important as its biochemical pathways. Remote though they may seem from the bedside, such studies are becoming of increasing importance to the practising physician, casting light on many unsolved clinical problems.

\section{REFERENCES}

Chance, B. \& Williams, G. R. (1956). Adv. Enzymol. r7, 65.

Gergely, J. (1966). A. Rev. Biochem. 35, 69r.

Gibson, K. \& Harris, P. (1970). Cardiovasc. Res. 4, 6.

Harris, P., Jones, J. H., Bateman, M., Chlouverakis, C. \& Gloster, J. (1964). Clin. Sci. $26,145$. Harris, P. \& Opie, L. (editors) (1971). Calcium and the Heart. London: Academic Press.

Huxley, H. E. (I959). Am. Heart F. 58, 777.

\section{EXPLANATION OF PLATE}

Electron micrograph of a longitudinal section through part of a rabbit myocardial cell. Magnification $\times 8000$. A very small part of an adjacent cell is shown in the lower left corner, where the two cell-wall membranes may be seen. $N$, nucleus; $M$, mitochondria; $Z, Z, Z$ lines embracing one sarcomere; $T \rightarrow$, transverse tubule; $\mathrm{L} \rightarrow$, longitudinal tubules. 